


\section{THE NATIONAL BUREAU OF STANDARDS}

\section{Functions and Activities}

The functions of the National Bureau of Standards are set forth in the Act of Congress, March 3, 1901, as amended by Congress in Public Law 619, 1950. These include the development and maintenance of the national standards of measurement and the provision of means and methods for making measurements consistent with these standards: the determination of physical constants and properties of materials; the development of methods and instruments for testing materials, devices, and structures; advisory services to Government Agencies on scientific and technical problems; invention and development of devices to serve special needs of the Government; and the development of standard practices, codes and specifications. The work includes basic and applied research, development, engineering, instrumentation, testing, evaluation, calibration services, and various consultation and information services. A major portion of the Bureau's work is performed for other Government Agencies, particularly the Department of Defense and the Atomic Energy Commission. The scope of activities is suggested by the listing of divisions and sections on the inside back cover.

\section{Reports and Publications}

The results of the Bureau's work take the form of either actual equipment and devices or published papers and reports. Reports are issued to the sponsoring agency of a particular project or program. Published papers appear either in the Bureau's own series of publications or in the journals of professional and scientific societies. The Bureau itself publishes three monthly periodicals, available from the Government Printing Office: The Journal of Research, which presents complete papers reporting technical investigations; the Technical News Bulletin, which presents summary and preliminary reports on work in progress; and Basic Radio Propagation Predictions, which provides data for determining the best frequencies to use for radio communications throughout the world. There are also five series of nonperiodical publications: The Applied Mathematics Series, Circulars, Handbooks, Building Materials and Structures Reports, and Miscellaneous Publications. 


\section{NATIONAL BUREAU OF STANDARDS REPORT}

NBS PROJECT

NBS REPORT

$7900-60-0079$

5594

STATISTICAL ANALYSIS OF A STATIONARY PROCESS

by

M. M. Siddiqui

NBS

U. S. DEPARTMENT OF COMMERCE

NATIONAL BUREAU OF STANDARDS

BOULDER LABORATORIES

Boulder, Colorado 


\section{IMPORTANT NOTICE}

NATIONAL BUREAU OF ments intended for use wi is subjected to additional tion, or open-literature lis mission is obtained in writi 25, D. C. Such permissior been specifically prepared
Approved for public release by the director of the National Institute of Standards and Technology (NIST) on October 9, 2015 or progress accounting docu:ports is formally published it lication, reprinting, reproducis not authorized unless pereau of Standards, Washington ency for which the Report has :opies for its own use. 


\begin{abstract}
The techniques developed in recent years to deal with stationary processes are presented in a manner suited to the purposes of those who use Fourier analysis or its variations for the analysis of their data. Necessary probability background is provided and the spectral analysis of discrete-time and continuous-time processes is carried out in detail with a number of examples to illustrate the applications of the theory.
\end{abstract}





\section{TABLE OF CONTENTS}

$\underline{\text { Page }}$

1. Introduction and summary $\ldots \ldots \ldots \ldots \ldots \ldots \ldots \ldots \ldots$

2. Some concepts in probability theory............. 4

3. Fourier analysis of a discrete-time process........ 8

4. Fourier analysis of a continuous-time process....... 15

5. Fourier analysis of a continuous-time process observed at discrete times............... 19

6. Linear filters $\ldots \ldots \ldots \ldots \ldots \ldots \ldots \ldots \ldots \ldots \ldots \ldots \ldots \ldots, 22$

7. Estimation of spectral density ............... 24

8. Examples......................... 27

References........................... 36 



\title{
STATISTICAL ANALYSIS OF A STATIONARY PROCESS
}

by

\author{
M. M. Siddiqui
}

\section{INTRODUCTION AND SUMMARY}

In recent years the approach to time series has undergone some important changes, and the methods of analysis have been modified accordingly.

The classical approach to time series may be summarized as follows. We have observations, $x_{1}, \ldots, x_{n}$, on a time variable and we represent

$$
x_{t}=m_{t}+\xi_{t}, \quad t=1,2, \ldots, n
$$

where $m_{t}$ is the deterministic and $\xi_{t}$ the random component of $x_{t}$ at time t. $\xi_{t}, t=1, \ldots, n$, are independent and identically distributed random variables, each with mean zero and variance $\sigma^{2}$. The distribution of $\xi_{t}$ is usually assumed to be normal and the variance $\sigma^{2}$ to be unknown. $m_{t}$ is represented either as a polynomial in $t$

$$
p(t)=c_{o}+c_{1} t+\ldots+c_{k} t^{k}
$$

where the degree of the polynomial, $k$, and the coefficients, $c_{0}, \ldots, c_{k}$, are assumed to be unknown constants; or $\mathrm{m}_{t}$ is represented as the sum of trigonometric terms 


$$
s(t)=a_{0}+a_{1} \cos \lambda_{1} t+b_{1} \sin \lambda_{1} t+\ldots+a_{p} \cos \lambda_{p} t+b_{p} \sin \lambda_{p} t
$$

where $p, a^{\prime} s, b^{\prime} s$ and $\lambda^{\prime} s$ are assumed to be unknown constants; or, more generally, $m_{t}$ is assumed to be the sum of $p(t)$ and $s(t)$. The method of least-squares is then employed to estimate the coefficients in any of these representations and the periodogram, i.e. $\left(a_{j}^{2}+b_{j}^{2}\right)$ multiplied by a constant, is used to test the significance of periods $2 \pi / \lambda_{j}$.

For a summary treatment of these methods the reader may refer to [10, Chapters 29, 30].

In more recent years, the concept of stationarity of time series has been playing an important role. We may still try to decompose a time series into a deterministic component, $m_{t}$, and a random component, $\xi_{t}$, if there are reasons to believe that some of the factors underlying our observations have been changing deterministically during the course of our observation. However, there are reasons to believe that in many physical experiments these factors are in statistical equilibrium, i. e. changing randomly with a fixed distribution function, during the course of our observation, or, even if they change more violently, this change is random rather than deterministic. In any case the deterministic component, $\mathrm{m}_{\mathrm{t}}$, is dropped, and a completely stochastic model takes its place. Several stochastic models have been developed to deal with non-stationary 
processes which represent certain phenomena, e.g. population growth, Brownian motion, epidemics, traffic on telephones and highways, servicing etc., under specified conditions. We will not describe them here.

In this report we will confine attention to the techniques developed in recent years to deal with stationary processes, i.e. time series under statistical equilibrium. These techniques are specially suited to analyse radio propagation data, taken over a short interval of time under more or less constant conditions. The material presented herein, excepting one or two original results, is explicitly or implicitly available elsewhere. However, it seemed desirable to present it here in a manner suited to the purposes of those who use Fourier analysis or its variations in dealing with time series data. It is interesting to note that the classical representation, $s(t)$, of a time series, still occupies a prominent place in the analysis, although the coefficients, $a^{\prime} s$ and b's, are not considered constants any more but random variables.

In section 2 necessary probability background will be provided. In sections 3 and 4 the spectral analysis of discrete-time and continuous-time processes, respectively, will be dealt with. In section 5 the case of a discrete sample from a continuous-time process will be considered. Linear filters, the estimation of spectral 
densities, and some examples of stationary processes will be presented in sections 6,7 and 8 , respectively. The references at the end of the report include only such publications which are directly connected with the subject matter of this report. For a more complete bibliography the reader may consult the Bibliography at the end of [9].

\section{SOME CONCEPTS IN PROBABILITY THEORY}

The basic idea in statistical analysis is that of a sample space. A sample space, $\Omega$, may be defined as the set of all possible distinct observations, $\omega$, on a physical experiment, actual or hypothetical, repeated under essentially similar conditions. Any subset, S, of $\Omega$ is called the event S. An additively closed family, B, of subsets of $\Omega$ is constructed and a probability measure, $P$, is defined on $B$. These concepts are introduced as a theoretical foundation of probability theory. From a practical point of view, the usual frequency concept of the probability of an event will be sufficient for proceeding further.

It may be that the observation on an experiment is a real number or a set of real numbers, e.g. observing the pressure and temperature of a given volume of a gas, in which case $\Omega$ may be taken an Euclidean space of proper dimensions, each characteristic being a coordinate. In certain other experiments the observation may not be expressed as a set of real numbers, e.g. observing the sex and color of hair of an 
individual, in which case we may associate real numbers to these characteristics under some conventional system, e.g. 0 for female and 1 for male. A random variable, $X(\omega)$, is, then, defined as a real-valued function of $\omega$, i.e., a function which associates a real number to every element $\omega$ of $\Omega$. A complex-valued function of $\omega$ will be called a complex-valued random variable. The subsets of $\Omega$ are associated through $X(\omega)$ with the subsets of the real line, $R_{1}$, and hence a probability measure is induced on $R_{1}$. If $S$ is a subset of $R_{1}$, we may speak of the probability that $X(\omega)$ belongs to $S$ and write $\operatorname{Pr}(X(\omega) \in S)$. In particular

$$
P(y)=\operatorname{Pr}(X(\omega) \leq y),
$$

where $y$ is a real number, is called the distribution function of $X(\omega)$. $P(y)$ is a non-decreasing function of its argument with $P(-\infty)=0$ and $P(\infty)=1$. It can be a step function or an absolutely continuous function or a mixture of both. In case $P(y)$ is absolutely continuous its derivative $P^{\prime}(y)=P(y)$ is called the probability density function or the frequency function of the random variable $X(\omega)$. Obviously

$$
p(y) \geq 0, \quad \operatorname{Pr}(X(\omega) \leq y)=P(y)=\int_{-\infty}^{y} p(x) d x, \int_{-\infty}^{\infty} p(y) d y=1
$$

The idea of a vector random variable presents no conceptual difficulty. In case of more than one random variable we speak of the joint distribution and the joint probability density functions. 
A random process, $\{x(t, \omega)\}$, besides being a function of $\omega$ is also a function of $t$. We will speak of $t$ as a time variable though it may be an arbitrary variable. For every value of $t, x(t, \omega)$ is a random variable. Thus a random process is a collection of random variables. For every fixed $\omega, x(t, \omega)$ is a sample function or realization of the process. Thus

$$
x(t, \omega)=A(\omega) \cos (\lambda(\omega) t+\phi(\omega))
$$

where $(A, \lambda, \phi)$ is a vector random variable, is a random process.

A random process may be visualized as a large number of simultaneous independent measurements of the same experiment over a long period of time. Theoretically the number of measurements is to be indefinitely large and the time of observation infinite in both directions. In practice we shall be dealing with a single realization of the process over a finite interval of time.

The time variable $t$ may vary continuously or may take only discrete values for a given random process. In the former case we shall call the process a continuous-time process and in the latter case, a discrete-time process. A continuous-time process may be made discrete by considering it at discrete time points.

There is no conceptual difficulty in talking about a vector random process nor in considering $t$ as a vector variable. In the 
following discussions, however, we will confine ourselves to a onedimensional random process and $t$ will always represent time. Furthermore, the letter $\omega$ will not occur explicitly and we will write $\{x(t)\}$ for a continuous-time process with $x(t)$ as a sample function and $\left\{x_{t}\right\}$ for a discrete-time process with $x_{t}$ as a sample function. If $\mathrm{u}$ is a random variable, Eu will be used to denote its mean value over the sample space $\Omega$.

A real random process, $\{x(t)\}$, will be called weakly $\underline{\text { stationary }}$ or stationary if, for all $t$ and $s$,

$$
\begin{aligned}
& \text { (i) } \operatorname{Ex}^{2}(t)<\infty \\
& \text { (ii) } \operatorname{Ex}(t)=\operatorname{Ex}(0) \\
& \text { (iii) } \operatorname{Ex}(t) x(t+s)=\operatorname{Ex}(0) x(s) \text {. }
\end{aligned}
$$

It will be called strictly stationary if the joint distribution of $x\left(t_{1}+h\right), \ldots, x\left(t_{n}+h\right)$ is identical with the joint distribution of $x\left(t_{1}\right), \ldots, x\left(t_{n}\right)$ for every $n, t_{1}, \ldots, t_{n}$ and every $h_{\text {. }}$

A process is called Gaussian if $x\left(t_{1}\right), \ldots, x\left(t_{n}\right)$ have a joint normal distribution for every $n, t_{1}, \ldots, t_{n}$. A Gaussian process is completely specified by $\operatorname{Ex}(t)$ and $\operatorname{Ex}(t) x(s)$ given for all $t$ and $s$. Hence a weakly stationary Gaussian process is strictly stationary. 


\section{FOURIER ANALYSIS OF A DISCRETE-TIME PROCESS}

Let $\left\{x_{t}\right\}$ be a stationary discrete-time process with $E x_{t}=m$ and $E\left(x_{t}-m\right)\left(x_{t+s}-m\right)=C_{s} \cdot C_{s}$ will be called the autocovariance function and $\rho_{S}=C_{S} / C_{O}$, the autocorrelation function of the process. Obviously, $C_{-s}=C_{s}, \rho_{-s}=\rho_{s}$. If $\mathrm{sC}_{\mathrm{S}} \rightarrow 0$ as $\mathrm{s} \rightarrow \infty$, then, the series

$$
\underset{s=-\infty}{\infty} C_{s} e^{-2 \pi i s \lambda}=C_{0}+2 \sum_{s=1}^{\infty} C_{s} \cos 2 \pi s \lambda
$$

is a Fourier series of its sum $f(\lambda)$, so that

$$
\begin{aligned}
& f(\lambda)=\sum_{s=-\infty}^{\infty} C_{s} e^{-2 \pi i s \lambda}=C_{0}+2 \sum_{s=1}^{\infty} C_{s} \cos 2 \pi s \lambda \\
& C_{s}=\int_{-1 / 2}^{1 / 2} f(\lambda) e^{2 \pi i s \lambda} d \lambda=\int_{-1 / 2}^{1 / 2} f(\lambda) \cos 2 \pi s \lambda d \lambda .
\end{aligned}
$$

We readily see from (1) that $f(-\lambda)=f(\lambda)$ and from (2) that $\int^{1 / 2} f(\lambda) d \lambda=C_{0} \cdot \quad f(\lambda)$ is called the power spectral density function $-1 / 2$

of the process $\left\{x_{t}\right\}$.

Consider the periodogram

(3) $I_{n}(\mu) \equiv \frac{1}{n}\left|\sum_{t=1}^{n}\left(x_{t}-m\right) e^{2 \pi i t \mu}\right|^{2}=\frac{1}{n} \sum_{k=1}^{n} \sum_{t=1}^{n}\left(x_{t}-m\right)\left(x_{k}-m\right) e^{2 \pi i(t-k)_{\mu}}$. 
We have $I_{n}(-\mu)=I_{n}(\mu)$ and

(4) $E I_{n}(\mu)=\frac{1}{n} \sum_{k=1}^{n} \sum_{t=1}^{n} C_{k-t} e^{2 \pi i(t-k) \mu}=\sum_{s=-(n-1)}^{n-1}\left(1-\frac{|s|}{n}\right) C_{s} e^{-i 2 \pi s \mu}$.

If $\sum_{1}^{\infty} \mathrm{sC}_{\mathrm{s}}<\infty$, we have

$$
\lim _{n \rightarrow \infty} \operatorname{EI}_{n}(\mu)=\sum_{-\infty}^{\infty} C_{s} e^{-i 2 \pi s \mu}=f(\mu)
$$

Since $I_{n}(\mu) \geq 0, \quad f(\mu) \geq 0$

Thus we may summarize the properties of $f(\lambda)$ in the following manner:

(i) $f(-\lambda)=f(\lambda)$ i.e. $f(\lambda)$ is symmetric,

(ii) $f(\lambda) \geq 0 \quad$ i.e. $f(\lambda)$ is non-negative,

(iii) $\int^{1 / 2} f(\lambda) d \lambda=C_{0}$;

$-1 / 2$

so that $f(\lambda) / C_{0}$ is a symmetrical frequency function over $(-1 / 2,1 / 2)$. We will set $f(\lambda)=0$ outside the interval $(-1 / 2,1 / 2)$.

\section{Writing}

(6)

$$
F(\lambda)=\int_{-1 / 2}^{\lambda} f(\mu) d \mu, \quad-1 / 2 \leq \lambda \leq 1 / 2,
$$


we will call $F(\lambda)$ the (power) spectral distribution function of $\left\{x_{t}\right\}$. $f *(\lambda)=f(\lambda) / C_{0}$ and $F *(\lambda)=f(\lambda) / C_{0}$ will be called the normalized spectral density and the normalized spectral distribution functions, respectively. Since $f(\lambda)$ is the Fourier series transformation of $C_{s}, f *(\lambda)$ is the Fourier series transformation of $\rho_{S}$. Thus we have proved the following theorem.

Theorem 1. If the Fourier series transformation, $f *(\lambda)$, of the autocorrelation function, $\rho_{s}$, of a discrete-time real stationary process exists, then it is a symmetrical frequency function over the interval $(-1 / 2,1 / 2)$.

It can also be shown that given a symmetrical frequency function, $f *(\lambda)$, over $(-1 / 2,1 / 2)$, we can find a real discrete-time stationary process, $\left\{x_{t}\right\}$, such that the Fourier coefficients of $f *(\lambda)$ are the autocorrelations of the process $\left\{x_{t}\right\}$.

Returning to the equation (5) we observe that if we have a sample $\mathrm{x}_{1}, \ldots, \mathrm{x}_{\mathrm{n}}$ then the periodogram $\mathrm{I}_{\mathrm{n}}(\lambda)$ is an asymptotically unbiased estimator of $f(\lambda)$. However, if we insert the value of $C_{s}$ from (2) into (4), we obtain

$$
\begin{aligned}
f_{n}(\mu) \equiv E I_{n}(\mu) & =\sum_{s=-(n-1)}^{n-1}\left(1-\frac{|s|}{n}\right) \int_{-1 / 2}^{1 / 2} e^{2 \pi i s(\lambda-\mu)} f(\lambda) d \lambda \\
& =\int_{-1 / 2}^{1 / 2} f(\lambda)\left[\sum_{s=-(n-1)}^{n-1}\left(1-\frac{|s|}{n}\right) e^{2 \pi i s(\lambda-\mu)}\right] d \lambda .
\end{aligned}
$$


Now, using the identities

$$
\sum_{s=1}^{p} z^{s}=\frac{z-z^{p+1}}{1-z} ; \quad \sum_{s=1}^{p} s z^{s}=\frac{z-(p+1) z^{p+1}+p z^{p+2}}{(1-z)^{2}}
$$

with $p=n-1$, and $z=e^{ \pm 2 \pi i(\lambda-\mu)}$, we can sum the series in the brackets and, using certain trigonometrical identities, we finally obtain

(7) $f_{n}(\mu)=\int_{-1 / 2}^{1 / 2} f(\lambda) \frac{\sin ^{2} \pi n(\lambda-\mu)}{n \sin ^{2} \pi(\lambda-\mu)} d \lambda=\frac{1}{n^{2}} \int_{n(\mu-1 / 2)}^{n(\mu+1 / 2)} f\left(\mu-\frac{v}{n}\right) \frac{\sin ^{2} \pi \nu}{\sin ^{2}(\pi v / n)} d \nu$.

Hence $I_{n}(\mu)$ is an unbiased estimate of a 'filtered' spectral density $f_{n}(\mu)$ for finite $n$. Only when $n \rightarrow \infty, f_{n}(\mu) \rightarrow f(\mu)$.

For convenience we suppose that $n=2 N+1$ and represent the sample $x_{1}, \ldots, x_{n}$ by the trigonometric series

$$
x_{t}=a_{o}+\sum_{j=1}^{N}\left(a_{j} \cos \frac{2 \pi j t}{n}+b_{j} \sin \frac{2 \pi j t}{n}\right), t=1,2, \ldots, n .
$$

The coefficients a's and b's are determined by the usual method of harmonic analysis, i.e., by the relations

$$
\begin{aligned}
& a_{0}=\frac{1}{n} \Sigma x_{t} \equiv \bar{x}, \\
& a_{j}=\frac{2}{n} \Sigma x_{t} \cos \frac{2 \pi j t}{n}=\frac{2}{n} \Sigma\left(x_{t}-c\right) \cos \frac{2 \pi j t}{n}, j=1, \ldots, N, \\
& b_{j}=\frac{2}{n} \Sigma x_{t} \sin \frac{2 \pi j t}{n}=\frac{2}{n} \Sigma\left(x_{t}-c\right) \sin \frac{2 \pi j t}{n}, j=1, \ldots, N,
\end{aligned}
$$


where the summations are with respect to $t$ from 1 to $\mathrm{n}$ and where c is an arbitrary constant. Thus, setting $c=m$, we have

$$
\begin{aligned}
\frac{n}{4}\left(a_{j}^{2}+b_{j}^{2}\right) & =\frac{1}{n}\left(\Sigma\left(x_{t}-m\right) \cos \frac{2 \pi j t}{n}\right)^{2}+\frac{1}{n}\left(\Sigma\left(x_{t}-m\right) \sin \frac{2 \pi j t}{n}\right)^{2} \\
& =\frac{1}{n}\left|\Sigma\left(x_{t}-m\right) e^{\frac{i 2 \pi j t}{n}}\right|^{2}=I_{n}\left(\frac{j}{n}\right), j=1, \ldots, N
\end{aligned}
$$

However, $I_{n}(0)=\frac{1}{n}\left|\Sigma\left(x_{t}-m\right)\right|^{2} \equiv n\left(a_{0}-m\right)^{2}$, and depends on the knowledge of the process mean $m$. If we replace $m$ by $\bar{x}$ everywhere, $I_{n}\left(\frac{j}{n}\right)$ will not change for $j=1, \ldots, N$, but $I_{n}(0) \equiv 0$ and no estimate at zero frequency will be available. It seems desirable, therefore, to estimate the process mean, $m$, by a much longer sample preferably from the observations which do not include $\mathrm{x}_{1}, \ldots, \mathrm{x}_{\mathrm{n}}$

Thus the representation (8) provides the periodogram estimate of the spectral density function at the frequencies $j / n, j=1, \ldots, N$, in terms of the coefficients $a_{j}$ and $b_{j}$.

We have

$$
\begin{aligned}
\operatorname{Ea} & =m, \\
\operatorname{var} a_{0}=E\left(a_{0}-m\right)^{2} & =\frac{1}{n^{2}} E\left|\Sigma\left(x_{t}-m\right)\right|^{2}=\frac{1}{n} E I_{n}(0) \\
& =\frac{1}{n} f_{n}(0) .
\end{aligned}
$$


Also, for $\mathrm{j} \neq 0$,

$$
\begin{aligned}
& E a_{j}=E b_{j}=0 \\
& \operatorname{var} a_{j}=E a_{j}^{2}=\frac{4}{n^{2}} E \sum_{t=1}^{n} \sum_{s=1}^{n}\left(x_{t}-m\right)\left(x_{s}-m\right) \cos \frac{2 \pi j t}{n} \cos \frac{2 \pi j s}{n} \\
& =\frac{2}{n^{2}} \sum_{t=1}^{n} \sum_{s=1}^{n} C_{t-s}\left[\cos \frac{2 \pi j(t-s)}{n}+\cos \frac{2 \pi j(t+s)}{n}\right] \\
& =\frac{2}{n} \underset{r=-(n-1)}{\sum-1}\left(1-\frac{|r|}{n}\right) C_{r} \cos \frac{2 \pi j r}{n}+\frac{2}{n^{2}} C_{o} \sum_{s=1}^{n} \cos \frac{4 \pi j s}{n} \\
& +\frac{4}{n^{2}} \sum_{r=1}^{n-1} \sum_{s=1}^{n-r} C_{r} \cos \frac{2 \pi j(2 s+r)}{n} \text {. }
\end{aligned}
$$

The first summation is seen to be equal to $2 / n E_{n}(j / n)=2 / n f_{n}(j / n)$. Using the trigonometrical identity

$$
\sum_{s=1}^{p} \cos (a+2 s \beta)=\operatorname{cosec} \beta \sin (p \beta) \cos \{a+(p+1) \beta\}
$$

with $\beta=2 \pi j / n, \quad a=2 \pi j r / n$, we find

$$
\sum_{s=1}^{n-r} \cos \frac{2 \pi j(2 s+r)}{n}=-\cot \frac{2 \pi j}{n} \sin \frac{2 \pi j r}{n} .
$$

Hence for $\mathrm{j} \neq 0$,

$$
\begin{aligned}
\operatorname{var} a_{j} & =\frac{2}{n} f_{n}\left(\frac{j}{n}\right)-\frac{4}{n^{2}} \cot \frac{2 \pi j}{n} \sum_{r=1}^{n-1} C_{r} \sin \frac{2 \pi j r}{n} \\
& =\frac{2}{n} f_{n}\left(\frac{j}{n}\right)+O\left(\frac{1}{n^{2}}\right), \text { since } \sum_{r=1}^{\infty} C_{r} \sin \frac{2 \pi j r}{n}<\infty .
\end{aligned}
$$


It can be shown similarly that

$$
\begin{aligned}
& \operatorname{var} b_{j}=\frac{2}{n} f_{n}\left(\frac{j}{n}\right)+\frac{4}{n^{2}} \cot \frac{2 \pi j}{n} \sum_{r=1}^{n-1} C_{r} \sin \frac{2 \pi j r}{n} \\
&=\frac{2}{n} f_{n}\left(\frac{j}{n}\right)+O\left(\frac{1}{n^{2}}\right), \\
& E a_{i} a_{j}=O\left(\frac{1}{n^{2}}\right), \quad E b_{i} b_{j}=O\left(\frac{1}{n^{2}}\right), \quad i \neq j, \\
& E a_{i} b_{j}=O\left(\frac{1}{n^{2}}\right), \quad \text { all } i, j .
\end{aligned}
$$

Thus the correlation between any two coefficients is of order $1 / n$. Now, if $\left\{x_{t}\right\}$ is a Gaussian process, $a_{j}$ and $b_{j}$ will be normally distributed and since different coefficients are approximately uncorrelated, they may be assumed to be independent of each other. Thus the $I_{n}(j / n)$ are also approximately independent of each other and

$$
2 I_{n}(j / n) / f_{n}(j / n)
$$

is approximately a $\chi^{2}$ variate with 2 degrees of freedom. We, therefore, have

$$
\operatorname{var} I_{n}(j / n) \cong f_{n}^{2}(j / n), j=1, \ldots, N
$$

Even if the process is not Gaussian, the result (13) can be proved to be valid. (13) shows that the periodogram, $I_{n}(\lambda)$, has standard error equal to (in fact a little greater than) $\mathrm{EI}_{\mathbf{n}}(\lambda)$, which makes it an undesirable estimate. 
We will discuss some proposed modifications of $I_{n}(\lambda)$ in section 7 .

4. FOURIER ANALYSIS OF A CONTINUOUS-TIME PROCESS

Let $\{x(t)\}$ be a continuous-time real stationary process with $E x(t)=m$ and $E[x(t)-m][x(t+s)-m]=C(s), \rho(s)=C(s) / C(0)$. If $\rho(s)$ is continuous at $s=0$ then it can be shown that it is continuous for all $s$ and also that

$$
\lim _{s \rightarrow 0} E|x(t+s)-x(t)|^{2}=0
$$

Consider the periodogram

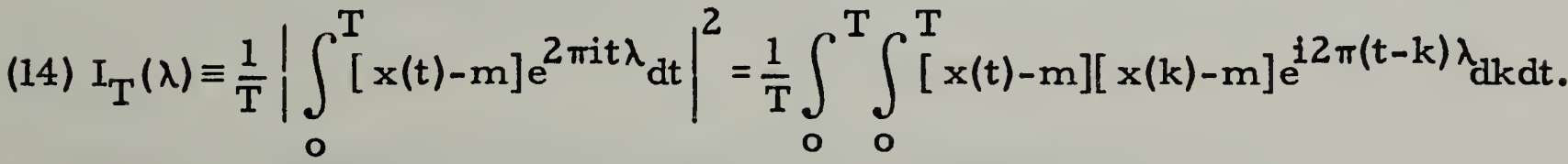

We have

(15) $g_{T}(\lambda) \equiv E I_{T}(\lambda)=\frac{1}{T} \int_{0}^{T} \int_{0}^{T} C(k-t) e^{i 2 \pi(t-k) \lambda} d k d t=\int_{-T}^{T}\left(1-\frac{|s|}{T}\right) C(s) e^{-i 2 \pi s \lambda} d s$.

Since $I_{T}(\lambda) \geq 0$, it follows that

$$
\int_{0}^{T} \int_{0}^{T} \rho(k-t) e^{i 2 \pi(t-k) \lambda} d k d t \geq 0
$$

Since $|\rho(s)| \leq 1, \rho(0)=1, \rho(s)$ is continuous and symmetric and the integral in $(16)$ is non-negative, therefore $[5, \mathrm{p} .91]$ we have the following theorem. 
Theorem 2. If the autocorrelation function $\rho(s)$ of a continuoustime real stationary process is continuous at $s=0$, it is the characteristic function of a symmetric distribution, i.e.

$$
\rho(s)=\int_{-\infty}^{\infty} e^{2 \pi i s \lambda} d G *(\lambda)
$$

where $G^{*}(\lambda)$ is a distribution function of a symmetric distribution over $(-\infty, \infty)$.

Conversely, it can be shown that for a symmetric distribution $\mathrm{dG} *(\lambda)$ the re exists a real continuous-time stationary process such that the characteristic function of $d G *(\lambda)$ is identical with the autocorrelation function of the process.

If $G *(\lambda)$ is absolutely continuous and has a frequency function $g *(\lambda)=d G * / d \lambda$, then it follows that

$$
g *(\lambda)=\int_{-\infty}^{\infty} \rho(s) e^{-2 \pi i s \lambda} d s
$$

and that $g *(\lambda)$ is continuous. Writing $g(\lambda)=C(0) g *(\lambda)$, we have the transform pair

$$
\begin{aligned}
& C(s)=\int_{-\infty}^{\infty} e^{2 \pi i s \lambda} g(\lambda) d \lambda \\
& g(\lambda)=\int_{-\infty}^{\infty} e^{-2 \pi i s \lambda} C(s) d s .
\end{aligned}
$$


Since $C(-s)=C(s)$, we have $g(-\lambda)=g(\lambda)$; also $g(\lambda) \geq 0$ as $g *(\lambda)$ is a frequency function, and

$$
\int_{-\infty}^{\infty} g(\lambda) d \lambda=C(0)
$$

$g(\lambda)$ is called the power spectral density function of the process $\{x(t)\}$. If $\int_{0}^{\infty} s C(s) d s$ exists, then from (15) it follows that

$$
\lim _{T \rightarrow \infty} g_{T}(\lambda)=g(\lambda)
$$

However, if we insert the value of $C(s)$ from (19) into (15), we obtain

$$
\begin{aligned}
g_{T}(\mu) & =\int_{-T}^{T} \int_{-\infty}^{\infty}\left(1-\frac{|s|}{T}\right) g(\lambda) e^{i 2 \pi(\lambda-\mu) s} d \lambda d s \\
& =\int_{-\infty}^{\infty} g(\lambda) d \lambda \int_{-T}^{T}\left(1-\frac{|s|}{T}\right) e^{i 2 \pi(\lambda-\mu) s} d s \\
& =\int_{-\infty}^{\infty} g(\lambda) \frac{\sin ^{2} \pi T(\lambda-\mu)}{\pi^{2} T(\lambda-\mu)^{2}} d \lambda \\
& =\int_{0}^{\infty}\left[g\left(\mu+\frac{\nu}{T}\right)+g\left(\mu-\frac{\nu}{T}\right)\right] \frac{\sin ^{2} \pi \nu}{\pi^{2} \nu^{2}} d \nu .
\end{aligned}
$$

Thus, if we have a continuous sample over $(0, T)$, then the periodogram, $I_{T}(\mu)$, is an unbiased estimate of $g_{T}(\mu)$ which is a 'filtered' spectral density. 
Now, the sample function over $(0, T)$ can be represented by a Fourier series, i.e.,

$$
x(t)=a_{o}+\sum_{j=1}^{\infty}\left(a_{j} \cos \frac{2 \pi j t}{T}+b_{j} \sin \frac{2 \pi j t}{T}\right), 0<t<T .
$$

We then have

$$
\begin{aligned}
& a_{0}=\frac{1}{T} \int_{0}^{T}(x(t) d t=\bar{x}, \\
& a_{j}=\frac{2}{T} \int_{0}^{T} x(t) \cos \frac{2 \pi j t}{T} d t=\frac{2}{T} \int_{0}^{T}[x(t)-c] \cos \frac{2 \pi j t}{T} d t, j \neq 0, \\
& b_{j}=\frac{2}{T} \int_{0}^{T} x(t) \sin \frac{2 \pi j t}{T} d t=\frac{2}{T} \int_{0}^{T}[x(t)-c] \sin \frac{2 \pi j t}{T} d t,
\end{aligned}
$$

where $c$ is an arbitrary constant. Setting $c=m$, we have

$$
\frac{T}{4}\left(a_{j}^{2}+b_{j}^{2}\right)=\frac{1}{T}\left|\int_{0}^{T}[x(t)-m] e^{\frac{i 2 \pi j t}{T}} d t\right|^{2}=I_{T}\left(\frac{j}{T}\right), j \neq 0 .
$$

Thus the representation (22) provides the periodogram estimate of $g_{T}(\lambda)$ at the frequencies $\lambda=j / T, j=1,2, \ldots$ in terms of the Fourier coefficients $a_{j}$ and $b_{j}$ and from (23) we see that these estimates are independent of the process mean $\mathrm{m}$. However,

$$
I_{T}(0)=\frac{1}{T}\left|\int_{0}^{T}[x(t)-m] d t\right|^{2}=T\left(a_{0}-m\right)^{2}
$$


and depends on the knowledge of $m$. If we replace $m$ by $\bar{x}$ then $I_{T}(0)=0$; hence, for an estimate at $\lambda=0$, we require the knowledge of $\mathrm{m}$ independent of the sample mean.

With calculations similar to those carried out for the discretetime case [see also 12 pp. 157-160] we can verify that

$$
\begin{aligned}
& E a_{0}=m, \quad E a_{j}=E b_{j}=0, j \neq 0, \\
& \operatorname{var} a_{0}=\frac{1}{T} g_{T}(0), \\
& \operatorname{var} a_{j}=\frac{2}{T} g_{T}\left(\frac{j}{T}\right)+O\left(\frac{1}{T^{2}}\right) \\
& \operatorname{var} b_{j}=\frac{2}{T} g_{T}\left(\frac{j}{T}\right)+O\left(\frac{1}{T^{2}}\right) \\
& E a_{i} a_{j}=O\left(\frac{1}{T^{2}}\right), \quad E b_{i} b_{j}=O\left(\frac{1}{T^{2}}\right), \quad i \neq j, \\
& E a_{i} b_{j}=O\left(\frac{1}{T^{2}}\right), \quad a 11 i, j .
\end{aligned}
$$

Also

$$
\operatorname{var} I_{T}(\lambda) \cong g_{T}^{2}(\lambda), \quad \lambda=j / T, \quad j \neq 0
$$

\section{FOURIER ANALYSIS OF A CONTINUOUS-TIME PROCESS OBSERVED AT DISCRETE TIMES}

The most interesting case is one in which we have a continuous record over $(0, T)$ as in section 4 but select a systematic sample $x(a), x(a+\delta), x(a+2 \delta), \ldots, x(a+\overline{n-1} \delta)$, where $0 \leq a<a+\overline{n-1} \delta \leq T$. 
We may then consider two processes $\{x(t)\}$ and $\left\{x_{t}\right\}$ where $x_{t}=x(a+\overline{t-1} \delta), t=0, \pm 1, \pm 2, \ldots$ We therefore have $C_{t}=C(t \delta)$. If $f(\lambda)$ and $g(\lambda)$ are the spectral densities of $\left\{x_{t}\right\}$ and $\{x(t)\}$ respectively, we have

$$
\begin{aligned}
\int_{1 / 2}^{1 / 2} f(\lambda) e^{i 2 \pi t \lambda} d \lambda & =\int_{-\infty}^{\infty} g(\lambda) e^{i 2 \pi t \delta \lambda} d \lambda \\
& =\frac{1}{\delta} \sum_{r=-\infty}^{\infty} \int_{r-1 / 2}^{r+1 / 2} g\left(\lambda^{\prime} / \delta\right) e^{i 2 \pi t \lambda^{\prime}} d \lambda^{\prime} \\
& =\int_{-1 / 2}^{1 / 2} e^{i 2 \pi t \lambda}\left[\frac{1}{\delta} \underset{r=-\infty}{\Sigma} g\left(\frac{\lambda+r}{\delta}\right)\right] d \lambda
\end{aligned}
$$

for every integer t. From the uniqueness of Fourier coefficients it follows that

$$
f(\lambda)=\frac{1}{\delta} \sum_{r=-\infty}^{\infty} g\left(\frac{\lambda+r}{\delta}\right),-1 / 2 \leq \lambda \leq 1 / 2 .
$$

The frequencies

$$
\frac{\lambda \pm 1}{\delta}, \frac{\lambda \pm 2}{\delta}, \ldots
$$

are called aliases to $\lambda / \delta$ as they become indistinguishable from $\lambda / \delta$ when we consider the discrete-time process $\left\{x_{t}\right\}$ instead of the continuous time process $\{x(t)\}$. 
If we set $a=\delta$ and $n \delta=T$, we have

(28) $\frac{1}{n} f\left(\frac{j}{n}\right)=\frac{1}{T} \underset{r=-\infty}{\infty} g\left(\frac{j+n r}{T}\right)$

$$
=\frac{1}{T} g\left(\frac{j}{T}\right)+\frac{1}{T} \sum_{r=1}^{\infty}\left[g\left(\frac{j+n r}{T}\right)+g\left(\frac{j-n r}{T}\right)\right],-\frac{n}{2} \leq j \leq \frac{n}{2} .
$$

Since $\int_{0}^{\infty} g(x+\mu) d \mu$ exists for every $x$, we have

$$
K_{n}=\int_{n / T}^{\infty} g(x+\mu) d \mu \rightarrow 0 \quad \text { as } n \rightarrow \infty
$$

Now $1 / T \sum_{k=0}^{\infty} g\left(x+\frac{n+k}{T}\right)$ is an approximating Riemann sum to

the integral $\mathrm{K}_{\mathrm{n}}$ with $\Delta \mu=1 / \mathrm{T}$ and hence tends to zero as $\mathrm{n} \rightarrow \infty$. Therefore, if $\mathrm{n}$ is large, we have for small values of $\mathrm{j}$

$$
\frac{1}{n} f\left(\frac{j}{n}\right) \cong \frac{1}{T} g\left(\frac{j}{T}\right)
$$

In practice, when we are analysing a record with superposed undesired 'noise' such as due to recording or reading instrument we will select $\delta$ or $n$ in such a way that $g\left(\frac{n-1}{2 T}\right)$ is dominated by the noise spectral density at the frequency $(n-1) / 2 T$. Here, we are assuming that the noise spectral density is negligible as compared to the spectral density of our data in low frequency ranges. We are then not interested in the value of $g(\lambda)$ for $\lambda>(n-1) / 2 T$, or, perhaps, 
the values of $g(\lambda)$ for $\lambda>(n-1) / 2 T$ are considered negligible. In this situation the effect of aliasing will be negligible except at the point $\lambda=(n-1) / 2 T$, where it will make our estimate twice as large as it actually should be.

\section{LINEAR FILTERS}

If we write $a *(\lambda)$ for the Fourier transform of a function $a(t)$ and if

$$
a(t)=\int_{-\infty}^{\infty} b(t-u) c(u) d u
$$

then it is easy to show that

$$
a *(\lambda)=b *(\lambda) c *(\lambda)
$$

$a(t)$ is called the convolution of $b(t)$ and $c(t)$.

Let $\{x(t)\}$ and $\{z(t)\}$ be two continuous-time real stationary processes such that

$$
x(t)=\int_{-\infty}^{\infty} w(t-u) z(u) d u=\int_{-\infty}^{\infty} w(u) z(t-u) d u
$$

where $W(t)$ is a real function integrable over $(-\infty, \infty) . W(t)$ is called the linear filter, $z(t)$ the input and $x(t)$ the output of the filter $W(t)$. if $C_{x}(t)$ and $C_{z}(t)$ denote the autocovariance and $g_{x}(\lambda)$ and $g_{z}(\lambda)$ the spectral densities of the processes $\{x(t)\}$ and $\{z(t)\}$ 
respectively, and $W *(\lambda)$ is the Fourier transform of $W(t)$, then we have

$$
\begin{aligned}
C_{x}(s)=E x(t) x(t+s) & =E \int_{-\infty}^{\infty} \int_{-\infty}^{\infty} W(u) W(v) z(t-u) z(t+s-v) d u d v \\
& =\int_{-\infty}^{\infty} \int_{-\infty}^{\infty} W(u) W(v) C_{z}(s+u-v) d u d v \\
g_{x}(\lambda)=\int_{-\infty}^{\infty} e^{-2 \pi i s \lambda} C_{x}(s) d s & =\int_{-\infty}^{\infty} \int_{-\infty}^{\infty} \int_{-\infty}^{\infty} d^{-2 \pi i s \lambda} W(u) W(v) C_{z}(s-u-v) d u d v d s .
\end{aligned}
$$

Making the transformation $s^{\prime}=s+u-v, u=u, v=v$, we have

$$
\begin{aligned}
g_{x}(\lambda) & =\int_{-\infty}^{\infty} d^{-2 \pi i s^{\prime} \lambda} C_{z^{\prime}}\left(s^{\prime}\right) d s^{\prime} \int_{-\infty}^{\infty} e^{i 2 \pi u \lambda} w(u) d u \int_{-\infty}^{\infty} e^{-i 2 \pi v \lambda} W(v) d v \\
& =g_{z}(\lambda) \quad|W *(\lambda)|^{2}, \quad-\infty \leq \lambda \leq \infty .
\end{aligned}
$$

Now, if the filter is known and the spectral density of one of the processes is known, then the relation (30) gives the spectral density of the other process. It is sometimes possible to adjust the filter in such a way that the output $x(t)$ is approximately a white noise, i. e., has a constant spectral density over a wide frequency range. The use of such a filter on $z(t)$ is called the prewhitening of $z(t)$ and is a powerful method in obtaining a reliable estimate of its spectral density. 
If in (29) we replace $x(t)$ by $x_{t}$ etc. and integrals by summations, we obtain

$$
f_{x}(\lambda)=|W *(\lambda)|^{2} f_{z}(\lambda), \quad-1 / 2 \leq \lambda \leq 1 / 2,
$$

where, now,

$$
W *(\lambda)=\sum_{k=-\infty}^{\infty} W_{k} e^{-i 2 \pi k \lambda} .
$$

For further study on this subject see [12, Ch. 5].

\section{ESTIMATION OF SPECTRAL DENSITY}

The purpose of Fourier analysis of a stationary process is to construct an estimator of the true spectral density function which has at least two desirable properties of being unbiased and consistent. It turns out that no estimator of $g(\lambda)$ or $f(\lambda)$ exists which is unbiased for finite sample size, although asymptotically unbiased estimators are available. The periodogram is one such estimator. Unfortunately, the variance of the periodogram remains bounded from below no matter how large a sample we take, i. e., it is an inconsistent estimator of $g(\lambda)$ or $f(\lambda)$. We therefore try to construct some other estimators which, besides being asymptotically unbiased, are consistent. In fact, several such estimators have been proposed and a general method of 'filtering' the periodogram is available which produces such estimators. We will only mention two such modified periodogram estimators, one suggested by Bartlett and the other by Tukey. 
Assuming $E x_{t}=0$ and writing

(32)

$$
c_{k} \equiv \frac{\sum_{r=1}^{n-k} x_{r} x_{r+k}}{n-k}, \quad k=0,1, \ldots, n-1, c_{-k}=c_{k} \text {. }
$$

we observe that

(33) $\quad I_{n}(\lambda)=\sum_{k=-(n-1)}^{n-1}\left(1-\frac{|k|}{n}\right) c_{k} \cos 2 \pi k \lambda, \quad \lambda=\frac{j}{n}, j=0,1, \ldots, \frac{n-1}{2}$.

Here, $I_{n}(0)$ can only be obtained when the process mean is known to be zero. Otherwise, $I_{n}(0)$ will remain arbitrary depending on our estimate of the process mean.

Bartlett suggests [1, also 9, p. 146]

(34) $I_{n}^{(1)}(\lambda)=\sum_{k=-m}^{m}\left(1-\frac{|k|}{m}\right) c_{k} \cos 2 \pi k \lambda, \quad \lambda=\frac{j}{m}, j=0,1, \ldots, m-1$,

where $m=0(n)$ but $m \rightarrow \infty$ as $n \rightarrow \infty$. Here, the sample covariances of large order have been avoided as they are subject to great sampling fluctuations. It is easy to see that $\lim _{n} \operatorname{EI}_{n}^{(1)}(\lambda)=f(\lambda)$. $n \rightarrow \infty$

The variance is given by

$$
\operatorname{var} I_{n}^{(1)}(\lambda) \cong \begin{cases}\frac{2 m}{3 n} f^{2}(\lambda), & \lambda \neq 0 \\ \frac{4 m}{3 n} f^{2}(0), & \lambda=0\end{cases}
$$


Tukey has proposed [9, p. 149; also 2]

$$
\begin{gathered}
I_{n}^{(2)}(\lambda)=c_{0}+2 \sum_{k=1}^{m-1}\left(.46 \cos \frac{\pi k}{m}+.54\right) c_{k} \cos \pi k \lambda \\
+(.46 \cos \pi+.54) c_{m} \cos \pi m \lambda \\
\lambda=\frac{j}{m}, j=0,1, \ldots, m-1,
\end{gathered}
$$

as an estimate of $f(\lambda)$, where $m=0(n)$ but $m \rightarrow \infty$ as $n \rightarrow \infty$. $I_{n}^{(2)}(\lambda)$ is also an asymptotically unbiased estimator of $f(\lambda)$, and its variance is

$$
\operatorname{var} I_{n}^{(2)}(\lambda) \cong\left\{\begin{array}{l}
\frac{m}{n} f^{2}(\lambda)\left[(.46)^{2}+2(.54)^{2}\right], \lambda \neq 0 \\
\frac{2 m}{n} f^{2}(0)\left[(.46)^{2}+2(.54)^{2}\right], \lambda=0
\end{array}\right.
$$

Tukey has also suggested a method of setting confidence bands on $f(\lambda)$, by showing that $I_{n}{ }^{(2)}(\lambda)$ is approximately a $\chi^{2}$ distribution with approximately $2 \mathrm{n} / \mathrm{m}$ degrees of freedom, [2]. However, $I_{n}^{(2)}\left(\lambda_{1}\right)$ and $I_{n}^{(2)}\left(\lambda_{2}\right)$ are not independent if $\left|\lambda_{1}-\lambda_{2}\right| \leq 3 / m$, so that the total degrees of freedom do not exceed the available degrees of freedom n. If the sample mean is subtracted from data before any further analysis, then as we have seen $I_{n}(0) \equiv 0$. It is difficult to give any meaning to $I_{n}{ }^{(1)}(0)$ and $I_{n}{ }^{(2)}(0)$ in such a situation. 


\section{EXAMPLES}

(i) White noise. Let $\left\{\xi_{t}\right\}$ be a stationary process such that $\mathrm{E} \xi_{\mathrm{t}} \xi_{\mathrm{t}+\mathrm{s}} \equiv \mathrm{C}_{\mathrm{s}}=0$ if $\mathrm{s} \neq 0$. Then, from (1), we have

$$
f(\lambda)=C_{0} \equiv \sigma_{\xi}^{2}, \quad-1 / 2 \leq \lambda \leq 1 / 2
$$

A process which has a constant spectral density is called a white noise. Conversely, if $f(\lambda)=C,-1 / 2 \leq \lambda \leq 1 / 2$, we have from (2), $C_{0}=C, C_{s}=0, s \neq 0$.

If, besides being uncorrelated, $\xi_{t}$ and $\xi_{t+s}$ are independent for all $s \neq 0$, the discrete time process $\left\{\xi_{t}\right\}$ is called a pure white noise $[9, \mathrm{p} .42]$.

From equation (19) we observe that we cannot set $g(\lambda)=$ constant in a continuous time process as this would imply that $C(0)$ was unbounded. However, by allowing the existence of Dirac's delta function and its Fourier transform, some authors have extended the concept of white noise to continuous time processes. We may, however, set $g(\lambda) \cong$ constant over a wide frequency band in some cases without making $C(0)$ infinite.

(ii) Moving average and autoregressive processes. Consider

$$
x_{t}=a_{o} y_{t}+a_{1} y_{t-1}+\ldots+a_{p} y_{t-p}=\sum_{k=0}^{p} a_{k} y_{t-k} .
$$


where $x_{t}$ and $y_{t}$ are stationary processes and $a_{k}$ are real constants. Since $x_{t}$ is the convolution of $w_{t}$ and $y_{t}$, where

$$
\begin{aligned}
w_{t} & =a_{t} \text { if } 0 \leq t \leq p \\
& =0 \text { otherwise, }
\end{aligned}
$$

we obtain

$$
f_{x}(\lambda)=\left|\sum_{k=0}^{p} a_{k} e^{-i 2 \pi k \lambda}\right|^{2} f_{y}(\lambda)
$$

If $y_{t}$ is a white noise, $x_{t}$ is called a moving average process. On the other hand if $x_{t}$ is a white noise and $\sum_{k=0}^{p} a_{k}{ }^{p-k}=0$ has all the roots within the unit circle $|z|=1$ in complex plane, $y_{t}$ is called an autoregressive process of order $p$. In this case $x_{t}$ is independent of $y_{t-1}, y_{t-2}, \ldots[9$, p. 38].

Special cases. (a) Simple moving average. If we set $a_{k}=1 /(p+1), k=0,1, \ldots, p$, in (38) we obtain

$$
x_{t}=\frac{1}{p+1} \sum_{j=0}^{p} y_{t-j}
$$

If $y_{t}$ is a white noise, the above scheme is called a simple moving average scheme. Since

$$
\left|\underset{i=0}{\sum} e^{-i \omega}\right|=\left|\frac{1-e^{-i(p+1) \omega}}{1-e^{-i \omega}}\right|=\left|\frac{e^{-i(p+1) / 2}}{e^{-i \omega / 2}} \frac{\sin (p+1) \omega / 2}{\sin \omega / 2}\right|=\left|\frac{\sin (p+1) \omega / 2}{\sin \omega / 2}\right| \text {, }
$$

we have from (39) 


$$
f_{x}(\lambda)=\frac{1}{(p+1)^{2}} \frac{\sin ^{2}(p+1) \pi \lambda}{\sin ^{2} \pi \lambda} f_{y}(\lambda) .
$$

However, since $y_{t}$ is a white noise, $f_{y}(\lambda)=\sigma_{y}^{2}$ and from $(40)$

$$
f_{y}(\lambda)=\sigma_{y}^{2}=(p+1) \sigma_{x}^{2}, \quad-1 / 2 \leq \lambda \leq 1 / 2
$$

and hence

$$
f_{x}(\lambda)=\frac{\sin ^{2}(p+1) \pi \lambda}{(p+1) \sin ^{2} \pi \lambda} \sigma_{x}^{2},-1 / 2 \leq \lambda \leq 1 / 2
$$

In estimating $f_{x}(\lambda)$, then, we only estimate $\sigma_{x}^{2}$ by the usual method of estimating a variance.

(b) Markov scheme. We consider the first-order autoregressive scheme (Markov scheme) in the form

$$
y_{t}=\rho y_{t-1}+x_{t}
$$

where $|\rho|<1$, and $x_{t}$ is a white noise. From (39)

$f_{y}(\lambda)=f_{x}(\lambda) /\left|1-\rho e^{-i 2 \pi \lambda}\right|^{2}=\sigma_{x}^{2}\left(1-2 \rho \cos 2 \pi \lambda+\rho^{2}\right)^{-1}, \quad-1 / 2 \leq \lambda \leq 1 / 2$,

since $f_{x}(\lambda)=\sigma_{x}^{2}$. Since $x_{t}$ is independent of $y_{t-1}$, we obtain from (42)

$$
\sigma_{y}^{2}=\rho^{2} \sigma_{y}^{2}+\sigma_{x}^{2}
$$

or

$$
\sigma_{x}^{2}=\sigma_{y}^{2}\left(1-\rho^{2}\right) .
$$


Thus

$$
f_{y}(\lambda)=\sigma_{y}^{2}\left(1-\rho^{2}\right)\left(1-2 \rho \cos 2 \pi \lambda+\rho^{2}\right)^{-1},-1 / 2 \leq \lambda \leq 1 / 2 .
$$

$\sigma_{y}^{2}$ and $\rho$ are estimated by the sample variance and first serial correlation coefficient respectively.

(c) Yule scheme. Consider the second-order autoregressive scheme (Yule scheme) in the form

$$
y_{t}=a y_{t-1}-\beta y_{t-2}+x_{t}
$$

where $x_{t}$ is a white noise and the roots of $z^{2}-a z+\beta=0$ lie within the unit circle $|z|=1$ in the complex plane. From (39) we have

$$
f_{y}(\lambda)=\frac{f_{x}(\lambda)}{1+a^{2}+\beta^{2}-2 a(1+\beta) \cos 2 \pi \lambda+2 \beta \cos 4 \pi \lambda},-1 / 2 \leq \lambda \leq 1 / 2 .
$$

Now, since $x_{t}$ is independent of $y_{t-1}$ and $y_{t-2}$, multiplying the equation (44) by $y_{t-1}$ and $y_{t-2}$ respectively and taking expectations, we obtain

or

$$
\begin{gathered}
C_{1}=a C_{0}-\beta C_{1}, \quad C_{2}=a C_{1}-\beta C_{0}, \\
\rho_{1}=a-\beta \rho_{1}, \quad \rho_{2}=a \rho_{1}-\beta,
\end{gathered}
$$

so that

$$
a=\frac{\rho_{1}\left(1-\rho_{2}\right)}{1-\rho_{1}^{2}}, \quad \beta=\frac{\rho_{1}^{2}-\rho_{2}}{1-\rho_{1}^{2}}
$$


If $\rho$ denotes the multiple correlation coefficient between $y_{t}$ and $\left(y_{t-1}, y_{t-2}\right)$, it is given by

$$
\left(1-\rho^{2}\right)=\left(1-\rho_{1}^{2}\right)^{-1}\left|\begin{array}{lll}
1 & \rho_{1} & \rho_{2} \\
\rho_{1} & 1 & \rho_{1} \\
\rho_{2} & \rho_{1} & 1
\end{array}\right|
$$

With simple evaluation we then obtain

$$
\sigma_{x}^{2}=\sigma_{y}^{2}\left(1-\rho^{2}\right)
$$

Since $f_{\mathbf{x}}(\lambda)=\sigma_{\mathbf{x}}^{2},-1 / 2 \leq \lambda \leq 1 / 2$, we finally have

(47) $f_{y}(\lambda)=\frac{\sigma_{y}^{2}\left(1-\rho^{2}\right)}{1+a^{2}+\beta^{2}-2 a(1+\beta) \cos 2 \pi \lambda+2 \beta \cos 4 \pi \lambda},-1 / 2 \leq \lambda \leq 1 / 2$.

To estimate $\rho, a$, and $\beta$, we need estimates of $\sigma_{y}, \rho_{1}$ and $\rho_{2}$. These estimates are provided by the sample variance and the first and second serial correlation coefficients respectively.

(iii) Processes related through a differential equation. For continuous-time processes we may consider the differential equation

$$
a_{p} \frac{d^{p} y}{d t^{p}}+a_{p-1} \frac{d^{p-1} y}{d t^{p-1}}+\ldots+a_{o} y=x(t)
$$

To obtain a relationship between the spectral densities $g_{x}(\lambda)$ and $g_{y}(\lambda)$ in this and similar cases, we outline a technique suggested by Cramér $[3,4]$ of representing the process as a Fourier transform of an orthogonal set function. 
If $I_{1}$ and $I_{2}$ are two disjoint sets on a real line, a set function $z(I)$ is called orthogonal if

$$
E z\left(I_{1}\right) \overline{z\left(I_{2}\right)}=0
$$

Here $\bar{z}$ denotes the complex conjugate of $z$. Writing

$$
d z(\mu)=z(d \mu)
$$

Cramér shows that every stationary continuous-time process, $\{x(t)\}$, may be represented as

$$
x(t)=\int_{-\infty}^{\infty} e^{i 2 \pi t \lambda} d z_{x}(\lambda)
$$

where $z_{x}(\lambda)$ is an orthogonal set function with $E\left|d z_{x}(\lambda)\right|^{2}=d G_{x}(\lambda)$, and $G_{x}(\lambda)$ is the spectral distribution function. If $x(t)$ is real, then, also

$$
x(t)=\int_{-\infty}^{\infty} e^{-i 2 \pi t \mu \overline{d z}(\mu)}
$$

Thus

$$
\begin{aligned}
C_{x}(s)=E x(t) x(t+s) & =E \iint e^{-i 2 \pi t \lambda} e^{+i 2 \pi(t+s) \mu} \overline{d z_{x}(\lambda)} d z_{x}(\mu) \\
& =\int_{-\infty}^{\infty} e^{+i 2 \pi s \lambda} d G_{x}(\lambda)
\end{aligned}
$$

which is the same as (19) in the case $G_{X}(\lambda)$ is absolutely continuous, 
Returning to (48) let us represent

$$
\begin{aligned}
& y(t)=\int_{-\infty}^{\infty} e^{i 2 \pi t \lambda} d z_{y}(\lambda) \\
& x(t)=\int_{-\infty}^{\infty} e^{i 2 \pi t \lambda} d z_{x}(\lambda)
\end{aligned}
$$

Then, from (48)

$$
\int_{-\infty}^{\infty} e^{i 2 \pi t \lambda}\left[\sum_{k=0}^{p} a_{k}(2 \pi i \lambda)^{k}\right] d z_{y}(\lambda)=\int_{-\infty}^{\infty} e^{i 2 \pi t \lambda} d z_{x}(\lambda) .
$$

From the uniqueness of the Fourier transform, we have

$$
\left[\sum_{k=0}^{p} a_{k}(2 \pi i \lambda)^{k}\right] d z_{y}(\lambda)=d z_{x}(\lambda)
$$

Multiplying by the complex conjugates and taking expectations, we obtain

$$
\left|\sum_{k=0}^{p} a_{k}(2 \pi i \lambda)^{k}\right|^{2} d G_{y}(\lambda)=d G_{x}(\lambda)
$$

or

(50)

$$
g_{x}(\lambda)=\left|\sum_{k=0}^{p} a_{k}(2 \pi i \lambda)^{k}\right|^{2} g_{y}(\lambda)
$$

A representation similar to (49) for a discrete-time process, $\left\{x_{t}\right\}$, is 


$$
x_{t}=\int_{-1 / 2}^{1 / 2} e^{2 \pi i t \lambda} d z_{x}(\lambda)
$$

where, now, $E\left|d z_{x}(\lambda)\right|^{2}=d_{x}(\lambda), \quad E d z_{x}(\lambda) \overline{d z_{x}(\mu)}=0, \lambda \neq \mu$, and $F_{x}(\lambda)$ is the spectral distribution function of $\left\{x_{t}\right\}$.

(iv) Shot noise. Let us consider instants, $t_{k}$, distributed at random on the time axis according to a Poisson process of density $\beta>0$. Let $n\left(t_{2}^{\prime}-t_{1}^{\prime}\right)$ be the number of the se instants belonging to the interval $\left(t_{1}^{\prime}, t_{2}^{\prime}\right)$. Writing $d n(t)=n(d t)$, it is then known that

$$
E d n(t)=\beta d t, \quad E[d n(t)]^{2}=\beta d t, \quad E[\operatorname{dn}(t) \operatorname{dn}(s)]=0, \quad t \neq s,
$$

so that $\mathrm{n}(\cdot)$ is an orthogonal set function.

Consider the process

$$
x(t)=\sum_{t j \leq t} a\left(t-t_{j}\right)
$$

where $a(t)$ is a real function with $a(t)=0$ if $t<0$, and $a(t)$ is integ rable over $(0, \infty)$.

Equation (52) may also be written

$$
x(t)=\int_{-\infty}^{\infty} a(t-s) d n(s), \quad \operatorname{dn}(s)=n(d s)
$$


We have

$$
E x(t)=\beta \int_{-\infty}^{\infty} a(t-s) d s=\beta \int_{0}^{\infty} a(s) d s \equiv m, \text { say. }
$$

Also,

$$
\begin{aligned}
C(u)=E[x(t)-m][x(t+u)-m] & =\int_{-\infty}^{\infty} a(t-s) a(t+u-s) \operatorname{var} d n(s) \\
& =\beta \int_{-\infty}^{\infty} a\left(t^{\prime}\right) a\left(t^{\prime}+u\right) d t^{\prime} .
\end{aligned}
$$

The right hand side, except for the factor $\beta$, is the convolution of $a(u)$ and $a(-u)$. Hence, if $a *(\lambda)$ is the Fourier transform of $a(t)$, we obtain

$$
g_{x}(\lambda)=\beta|a *(\lambda)|^{2}
$$

Thus $x(t)$ is composed of a 'direct current' $\beta \int_{0}^{\infty} a(t) d t$ and a 'shot noise' with continuous spectral density $\beta|a *(\lambda)|^{2}$.

$$
\begin{aligned}
& \text { Special case. } \quad a(t)= \begin{cases}\frac{1}{T} e^{-t / T} & t \geq 0 \\
0 & t<0 .\end{cases} \\
& |a *(\lambda)|^{2}=\frac{1}{1+4 \pi^{2} \mathrm{~T}^{2} \lambda^{2}} \text {, } \\
& g_{x}(\lambda)=\frac{\beta}{1+4 \pi^{2} T^{2} \lambda^{2}} ; \quad E x=\beta, \operatorname{var} x=\frac{\beta}{2 T}
\end{aligned}
$$

For further examples see [12, chs. $3,4,5]$. 
1. Bartlett, M. S., "Periodogram analysis and continuous spectra," Biometrika, Vol. 37 (1950).

2. Blackmann, R. B., and Tukey, J. W., "The measurement of power spectra from the point of view of communications engineering," Parts I and $\amalg$, The Bell System Technical Journal, Vol. 37, Nos. 1, 2 (1958).

3. Cramér, Harald, "On the theory of stationary random processes," Annals of Mathematics, Vol. 41 (1940).

4. "On harmonic analysis in certain functional spaces," Ark. Mat. Astr. Fys., Vol. 28B (1942).

5. Mathematical Methods of Statistics, Princeton University Press, Princeton (1951).

6. Davis, R. C., "On the detection of sure signals in noise," Journal of Applied Physics, Vol. 25 (1954).

7. Fisher, R. A., "Tests of significance in harmonic analysis," Proceedings of Royal Society, London, Vol. A125 (1924).

8. Goldman, S., Frequency Analysis, Modulation and Noise, New York (1948).

9. Grenander, Ulf, and Rosenblatt, Murray, Statistical Analysis of Stationary Time Series, John Wiley \& Sons, New York (1957).

10. Kendall, M. G., The Advanced Theory of Statistics, Vol. II, Charles Griffin and Company Limited, London (1948).

11. Kolmogorov, A., "Stationary sequences in Hilbert space," Bull. Math. Univ. Moscow, Vol. 2, No. 6 (1941).

12. Laning, J. H., and Battin, R. H., Random Processes in Automatic Control, McGraw-Hill Book Company, Inc., New York (1956).

13. Mann, H. B. Introduction to the Theory of Stochastic Processes Depending on a Continuous Parameter, NBS Applied Maths. Series 24 (1953). 
14. Mann, H.B., and Wald, Abraham, "On the statistical treatment of linear stochastic difference equations," Econometrica, Vol. 11 (1943).

15. Maruyama, G., "The harmonic analysis of stationary stochastic processes," Mem. Fac. Sci. Kyusyu Uni., Ser. A, Vol. 4 (1949).

16. Parzen, Emanuel, "On consistent estimates of the spectrum of a stationary time series," Annals of Math. Stats., Vol. 28 (1957).

17. "On choosing an estimate of the spectral density function of a stationary time series," Annals of Math. Stats., Vol. 28 (1957).

18. Rice, S. O., "Mathematical analysis of random noise," reproduced in Selected Papers on Noise and Stochastic Processes, Dover Publications, New York (1954).

19. Wold, Herman, A Study in the Analysis of Stationary Time Series, Uppsala (1938).

20. Whittle, P., Hypothesis Testing in Time Series Analysis, Uppsala (1951). 

U. S. DEPARTMENT OF COMMERCE

Sinclair Weeks, Secretary

NATIONAL BUREAU OF STANDARDS

A. V. Astin, Director



\section{THE NATIONAL BUREAU OF STANDARDS}

The scope of the scientific program of the National Bureau of Standards at laboratory centers in Washington, D. C., and Boulder, Colorado, is given in the following outline:

Washington, D.C.

Electricity and Electronics. Resistance and Reactance. Electron Devices. Electrical Instruments. Magnetic Measurements. Dielectrics. Engineering Electronics. Electronic Instrumentation. Electrochemistry.

Optics and Metrology. Photometry and Colorimetry. Optical Instruments. Photographic Technology. Length. Engineering Metrology.

Heat. Temperature Physics. Thermodynamics. Cryogenic Physics. Rheology. Engine Fuels. Free Radicals.

Atomic and Radiation Physics. Spectroscopy. Radiometry. Mass Spectrometry. Solid State Physics. Electron Physics. Atomic Physics. Neutron Physics. Nuclear Physics. Radioactivity. X-rays. Betatron. Nucleonic Instrumentation. Radiological Equipment. AEC Radiation Instruments.

Chemistry. Organic Coatings. Surface Chemistry. Organic Chemistry. Analytical Chemistry. Inorganic Chemistry. Electrodeposition. Molecular Structure and Properties. Physical Chemistry. Thermochemistry. Spectrochemistry. Pure Substances.

Mechanics. Sound. Mechanical Instruments. Fluid Mechanics. Engineering Mechanics. Mass and Scale. Capacity, Density, and Fluid Meters. Combustion Controls.

Organic and Fibrous Materials. Rubber. Textiles. Paper. Leather. Testing and Specifications. Polymer Structure. Plastics. Dental Research.

Metallurgy. Thermal Metallurgy. Chemical Metallurgy. Mechanical Metallurgy. Corrosion. Metal Physics.

Mineral Products. Engineering Ceramics. Glass. Refractories. Enameled Metals. Concreting Materials. Constitution and Microstructure.

Building Technology. Structural Engineering. Fire Protection. Air Conditioning, Heating, and Refrigeration. Floor, Roof, and Wall Coverings. Codes and Safety Standards. Heat Transfer. Applied Mathematics. Numerical Analysis. Computation. Statistical Engineering. Mathematical Physics.

Data Processing Systems. SEAC Engineering Group. Components and Techniques. Digital Circuitry. Digital Systems. Analogue Systems. Application Engineering.

- Office of Basic Instrumentation

- Office of Weights and Measures

\section{Boulder, Colorado \\ BOULDER LABORATORIES \\ F. W. Brown, Director}

Cryogenic Engineering. Cryogenic Equipment. Cryogenic Processes. Properties of Materials. Gas Liquefaction.

Radio Propagation Physics. Upper Atmosphere. Ionosphere. Regular Propagation Services. Sun-Earth Relationships. VLF Research.

Radio Propagation Engineering. Data Reduction Instrumentation. Modulation Systems. Navigation Systems. Radio Noise. Tropospheric Measurements. Tropospheric Analysis. Radio Systems Application Engineering. Radio Meteorology.

Radio Standards. High Frequency Electrical Standards. Radio Broadcast Service. High Frequency Impedance Standards. Calibration Center. Microwave Physics. Microwave Circuit Standards. 


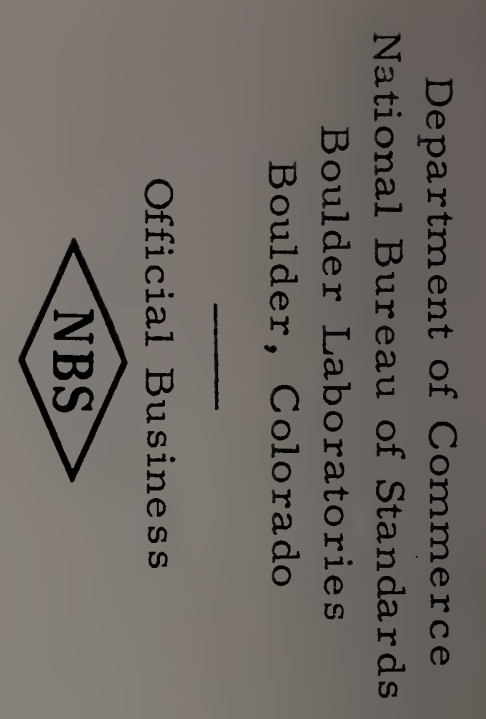



$\Omega$

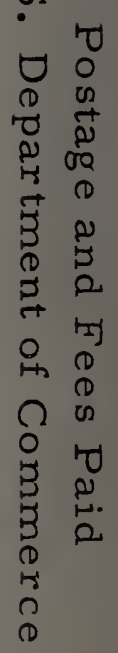

